\title{
The number of $P$-vertices in a matrix with maximum nullity
}

\author{
Rosário Fernandes ${ }^{\mathrm{a}, *, 1}$, Henrique F. da Cruz ${ }^{\mathrm{b}, 2}$ \\ a CMA and Faculdade de Ciências e Tecnologia, Universidade Nova de Lisboa, \\ 2829-516 Caparica, Portugal \\ b Universidade da Beira Interior, Centro de Matemática e Aplicações (CMA-UBI), \\ Rua Marquês D'Avila e Bolama, 6201-001 Covilhã, Portugal
}

\section{A R T I C L E I N F O}

\section{Article history:}

Received 7 April 2016

Accepted 16 February 2018

Available online 24 February 2018

Submitted by Bryan L.Shader

\section{$M S C$ :}

$15 \mathrm{~A} 18$

$15 \mathrm{~A} 48$

$05 \mathrm{C} 50$

Keywords:

Trees

Acyclic matrices

Maximum nullity

Parter vertices

\section{A B S T R A C T}

Let $T$ be a tree with $n \geq 2$ vertices. Set $\mathcal{S}(T)$ for the set of all real symmetric matrices whose graph is $T$. Let $A \in \mathcal{S}(T)$ and $i \in\{1, \ldots, n\}$. We denote by $A(i)$ the principal submatrix of $A$ obtained after deleting the row and column $i$. We set $m_{A}(0)$ for the multiplicity of the eigenvalue zero in $A$ (the nullity of $A$ ). When $m_{A(i)}(0)=m_{A}(0)+1$, we say that $i$ is a $P$-vertex of $A$. As usual, $M(T)$ denotes the maximum nullity occurring of $B \in \mathcal{S}(T)$. In this paper we determine an upper bound and a lower bound for the number of $P$-vertices in a matrix $A \in \mathcal{S}(T)$ with nullity $M(T)$. We also prove that if the integer $b$ is between these two bounds, then there is a matrix $E \in \mathcal{S}(T)$ with $b P$-vertices and maximum nullity.

() 2018 Elsevier Inc. All rights reserved.

\footnotetext{
* Corresponding author.

E-mail addresses: mrff@fct.unl.pt (R. Fernandes), hcruz@ubi.pt (H.F. da Cruz).

1 Rosário Fernandes is partially supported by the Fundação para a Ciência e a Tecnologia, Portugal, UID/MAT/00297/2013.

${ }^{2}$ Henrique F. da Cruz is partially supported by the Fundação para a Ciência e a Tecnologia, Portugal, UID/MAT/00212/2013.
} 


\section{Introduction}

Let $T=(X, \mathcal{E})$ be a tree with $n \geq 2$ vertices, where $X=\{1,2, \ldots, n\}$, and let $\mathcal{S}(T)$ be the set of all $n$-by- $n$ real symmetric matrices $A=\left[a_{i j}\right]$ whose graph is $T\left(a_{i j} \neq 0\right.$, with $i \neq j$, if and only if there is an edge between $i$ and $j$ ). No restriction is placed on the main diagonal. If $\alpha \subseteq\{1,2, \ldots, n\}$, then we denote the principal submatrix of $A \in \mathcal{S}(T)$ resulting from deletion (respectively, retention) of rows and columns in $\alpha$ by $A(\alpha)$ (respectively, $A[\alpha]$ ). When $\alpha$ consists of a single element $i$, instead of $A(\{i\})$ we simply write $A(i)$. Note that, if we denote by $T-\alpha$ the subgraph of $T$ after removing vertices in $\alpha$ and edges incident in at least one vertex of $\alpha$, then $A(\alpha)$ is a matrix whose graph is $T-\alpha$. Using a similar notation, when $\alpha=\{i\}$, we write $T-i$ instead of $T-\{i\}$. When $H$ is a subgraph of $T, A[H]$ denotes the principal submatrix of $A$ resulting from deletion of rows and columns that correspond to vertices not in $H$.

The multiplicity of $\lambda \in \mathbb{R}$ as an eigenvalue of $A \in \mathcal{S}(T)$ is denoted by $m_{A}(\lambda)$, and the spectrum of $A$ is denoted by $\sigma(A)$. As a consequence of the Cauchy interlacing theorem [7], we have

$$
m_{A}(\lambda)-1 \leq m_{A(i)}(\lambda) \leq m_{A}(\lambda)+1
$$

for all $1 \leq i \leq n$.

Consequently, we have three possibilities for a vertex removed in $T$ :

- In the case of equality in the right-hand side of (1), the element $i$ is known as a Parter vertex of $A$.

If $\lambda=0$, then the element $i$ (vertex $i$ of $T$ ) is known as a $P$-vertex of $A$.

- If $m_{A(i)}(\lambda)=m_{A}(\lambda)$, then $i$ is known as a neutral vertex of $A$.

- If $m_{A(i)}(\lambda)=m_{A}(\lambda)-1$, then $i$ is known as a downer vertex of $A$.

When a vertex removed in $T$ verifies $m_{A(i)}(\lambda) \geq m_{A}(\lambda)$, then $i$ is a Parter vertex or a neutral vertex of $A$, and it is known as an $F$-vertex of $A$.

Note that if $\lambda \neq 0$ and $\lambda$ is an eigenvalue of $A$, then zero is an eigenvalue of $B=A-\lambda I$ and $m_{B}(0)=m_{A}(\lambda)$. So, we can focus our study in the eigenvalue zero.

One may also derive from the right-hand of (1) that $m_{A(\alpha)}(0) \leq m_{A}(0)+|\alpha|$, for any nonempty subset $\alpha$ of $\{1,2, \ldots, n\}$. When we have $m_{A(\alpha)}(0)=m_{A}(0)+|\alpha|$, we call $\alpha$ a $P$-set of $A,[10]$. The existence of the $P$-vertices was stated in the next theorem, a generalization of the Parter-Wiener Theorem $[15,16]$. We denote by $d_{T}(v)$ the degree of a vertex $v$ of $T$.

Theorem 1.1. [10] Let $A$ be a Hermitian matrix whose graph is a tree $T$, and suppose that there exists a vertex $v$ of $T$ and a real number $\lambda$ such that $\lambda \in \sigma(A) \cap \sigma(A(v))$. Then

1. there is a vertex $v^{\prime}$ of $T$ such that $m_{A\left(v^{\prime}\right)}(\lambda)=m_{A}(\lambda)+1$; 
2. if $m_{A}(\lambda) \geq 2$, then $v^{\prime}$ may be chosen so that $d_{T}\left(v^{\prime}\right) \geq 3$, and there are at least three components $T_{1}, T_{2}$, and $T_{3}$ of $T-v^{\prime}$ such that $m_{A\left[T_{i}\right]}(\lambda) \geq 1, i=1,2,3$;

3. if $m_{A}(\lambda)=1$, then $v^{\prime}$ may be chosen so that $d_{T}\left(v^{\prime}\right) \geq 2$ and there are two components $T_{1}$ and $T_{2}$ of $T-v^{\prime}$ such that $m_{A\left[T_{i}\right]}(\lambda)=1, i=1,2$.

A characterization of $F$-vertices was stated in [14]:

Theorem 1.2. [14] Let $A$ be an $n$-by-n singular, symmetric matrix, and $i \in\{1, \ldots, n\}$. The following statements are equivalent:

1. The ith coordinate of every nullvector of $A$ is equal to zero;

2. The vertex $i$ is an $F$-vertex of $A$.

Many researchers $([2,3,10,11,13])$ have studied $P$-vertices and $P$-sets of matrices whose graph is a tree. In [2], the authors found an upper bound for the size of any P-set of any symmetric matrix of order $n$. They also characterized the trees where this upper bound is attained.

As usual, [9], $M(T)$ is the maximum multiplicity occurring for an eigenvalue of an $A \in \mathcal{S}(T)$.

The thrust of this paper is to provide an upper bound and a lower bound on the number of $P$-vertices of a matrix $A \in \mathcal{S}(T)$ with nullity $M(T)$. We also prove that if the integer $b$ is a number between these two bounds then there is a matrix $C \in \mathcal{S}(T)$ with $b$ $P$-vertices and maximum nullity. The structure of matrices with maximum nullity was studied in [11]. The following result is a combination of results that were stated there.

Theorem 1.3. [11] Suppose that $T$ is a tree, and $A \in \mathcal{S}(T)$ is such that $m_{A}(0)=M(T)$. Then

1. no vertex of $T$ is a neutral vertex of $A$,

2. the removal of a $P$-vertex of $A$ in $T$ does not change the status of any other vertex,

3. the each set of $P$-vertices of $A$ is a $P$-set of $A$.

The paper is organized as follows. In Section 2 we introduce some additional notation, study the problem when $T$ is a path and present some results useful in the rest of the paper. In Section 3 we define a family of subsets of vertices of the tree, $T$, necessary to find bounds of the number of $P$-vertices in a matrix $A \in \mathcal{S}(T)$ with maximum nullity. In Section 4 we prove results about the sets defined in Section 3, with smallest cardinality. The study about the sets with largest cardinality appears in Section 5. Section 6 is dedicated to establish a relation between some of the sets defined in Section 3, in a tree $T$, and a particular subtree of $T$. Finally, in Section 7 we present the bounds for the number of $P$-vertices of a matrix $A \in \mathcal{S}(T)$ with maximum nullity. 


\section{The number of $P$-vertices in a path}

In this section we studied the particular case of paths. We do this for motivation, and for the readers who may only be interested in paths. Many of the known results presented in this work can be found in a good book of spectral graph theory, besides the references. The path cover number, $P(T)$, of a tree $T$ is defined as the minimum number of vertex disjoint paths that cover all vertices of $T$. We will use the following fundamental theorem (see [9] or [6]):

Theorem 2.1. If $T$ is a tree then

$$
M(T)=P(T)
$$

When $T$ is a path, using last theorem, we conclude that

Corollary 2.2. Let $T$ be a path, $A \in \mathcal{S}(T)$ and $\lambda$ be an eigenvalue of $A$. Then $m_{A}(\lambda)=1$.

As noted in [10], if $T$ is a path with $n$ vertices, and $A \in \mathcal{S}(T)$, then $A$ is permutationally similar to an $n$-by- $n$ irreducible tridiagonal symmetric matrix. Consequently, by Corollary 2.2, any eigenvalue of $A$ has multiplicity one. Moreover, we have the classical fact.

Proposition 2.3. If $A$ is an $n$-by-n irreducible tridiagonal symmetric matrix, then the eigenvalues of $A(1)$ and $A(n)$ each strictly interlace those of $A$.

When $T$ is a tree, as usual, we denote by $L(T)$ the Laplacian matrix, i.e., the matrix $L(T)=A(T)-D(T)$ where $A(T)$ is the adjacency matrix of $T$ and $D(T)$ is a diagonal matrix with the degree of vertices of $T$. It is well known that zero is an eigenvalue of $L(T)$. Moreover, if $\lambda$ is an eigenvalue of $L(T)$ then $\lambda \geq 0$. The next result gives the multiplicity of the eigenvalue 0 of the Laplacian matrix of a path. It follows from general results on for graphs (see theorem 7.1.2 of [1]), and from results in [5]. We include a simple proof here for convenience.

Theorem 2.4. Let $T$ be a path. Let $L(T)$ be the Laplacian matrix of $T$. Then

$$
m_{L(T)}(0)=1=P(T)
$$

and there are no P-vertices of $L(T)$.

Proof. If $T$ is a path with $n$ vertices, then $P(T)=1$. Since $P(T)=1$ then $m_{L(T)}(0)=$ $1=P(T)$.

Let $\{1,2, \ldots, n\}$ be the set of vertices of $T$ with $i$ adjacent to $i-1$ and $i+1$, for $2 \leq i \leq n-1$. 
If $i=1$ or $i=n$, then by Proposition 2.3, $i$ is not a $P$-vertex of $L(T)$.

If $i \in\{2, \ldots, n-1\}$, consider the paths $T_{1}$ and $T_{2}$, where $T_{1}$ is the subpath of $T$ indexed by vertices $1, \ldots i-1, i$ and $T_{2}$ is the subpath of $T$ indexed by vertices $i, i+1, \ldots, n$. Since

$$
m_{L(T)(i)}(0)=m_{L\left(T_{1}\right)(i)}(0)+m_{L\left(T_{2}\right)(i)}(0),
$$

by Proposition 2.3, $i$ is not a $P$-vertex of $L(T)$.

So, we conclude that no vertex of $T$ is a $P$-vertex of $L(T)$.

The next result follows from general results in [12], concerning how the multiplicity of an eigenvalue can change when two vertices are deleted (see proposition 4.7). We give a self contained proof here.

Lemma 2.5. Let $T$ be a path and let $A \in \mathcal{S}(T)$ such that $m_{A}(0)=1$. If $x$ and $y$ are $P$-vertices of $A$ then $x$ is not adjacent to $y$.

Proof. Suppose that $x$ is adjacent to $y$. By Proposition 2.3 neither $x$ nor $y$ are terminal vertices of $T$. Let $T_{1}$ and $T_{2}$ be the two paths of $T-x$. If $A_{1}$ is the submatrix of $A(x)$ associated with $T_{1}$ and $A_{2}$ is the submatrix of $A(x)$ associated with $T_{2}$ then $A(x)=A_{1} \oplus$ $A_{2}, m_{A(x)}(0)=2$ and $m_{A_{1}}(0)=m_{A_{2}}(0)=1$. Suppose that $y$ is a vertex of $T_{1}$. Using $y$ instead of $x$, we conclude that $A(y)=B_{1} \oplus B_{2}, m_{A(y)}(0)=2$ and $m_{B_{1}}(0)=m_{B_{2}}(0)=1$, where $B_{1}$ and $B_{2}$ are the submatrices of $A(y)$ associated with the paths of $T-y$. Suppose that $x$ is a vertex of the path associated with $B_{1}$. By Proposition 2.3, $m_{B_{1}(x)}(0)=0$. This is impossible because $A_{2}=B_{1}(x)$.

As in [13], the number of $P$-vertices of $A \in \mathcal{S}(T)$ is denoted by $P_{v}(A)$.

Proposition 2.6. Let $T$ be a path with $n$ vertices and let $A \in \mathcal{S}(T)$ such that $m_{A}(0)=1$. Then

$$
0 \leq P_{v}(A) \leq\left\lfloor\frac{n-1}{2}\right\rfloor
$$

Proof. Using Proposition 2.3 we know that a $P$-vertex is not a terminal vertex of $T$. By Lemma 2.5 we know that there are not adjacent $P$-vertices. So, $P_{v}(A) \leq\left\lfloor\frac{n-1}{2}\right\rfloor$.

Theorem 2.7. Let $T$ be a path with $n$ vertices and let $b$ be an integer such that

$$
0 \leq b \leq\left\lfloor\frac{n-1}{2}\right\rfloor
$$

Then, there is a matrix $A \in \mathcal{S}(T)$ such that $m_{A}(0)=1$ and $P_{v}(A)=b$. 
Proof. Let $T$ be a path. If $b=0$, then by Theorem $2.4, L(G)$ is the sought matrix. Assume now that $b \geq 1$. Remove $b$ vertices $i_{1}, \ldots, i_{b}$ from $T$ such that none of the removed vertices is a terminal vertex of $T$, and no two of them are adjacent.

Let $P_{1}, \ldots, P_{b+1}$ be the paths of $T-\left\{i_{1}, \ldots, i_{b}\right\}$ such that $P_{1}$, and $P_{b+1}$ contain the terminal vertices of $T$. We denote the forest of the paths $P_{1}, \ldots, P_{b+1}$ by $G$. Let $A=$ $\left[a_{i, j}\right] \in \mathcal{S}(T)$ be a matrix such that $A\left(\left\{i_{1}, \ldots, i_{b}\right\}\right)=L(G)$ (the Laplacian matrix of $G$ ), and assuming that vertex $i_{t}, 1 \leq t \leq b$ is adjacent to vertices $j_{t}$ and $j_{t}^{\prime}, a_{i_{t}, j_{t}}=a_{j_{t}, i_{t}}=c$, for some $c \neq 0$, and $a_{i_{t}, j_{t}^{\prime}}=a_{j_{t}^{\prime}, i_{t}}=-c$.

As $m_{L(G)}(0)=b+1$, by the Cauchy interlacing Theorem and using Corollary 2.2, $m_{A}(0)=1$, and $i_{1}, \ldots, i_{b}$ are $P$-vertices of $A$.

Let $v$ be the $n$-by- 1 vector whose $j$ th coordinates, for $j \in\left\{i_{1}, \ldots, i_{b}\right\}$ are zero, and all the others coordinates are 1 . Then $v$ is a nullvector of $A$. We have proved that $A$ has a nullvector whose $j$ th coordinates, for all $j \in\{1, \ldots, n\}-\left\{i_{1}, \ldots, i_{b}\right\}$, are not zero. By Theorem 1.2, none of the vertices of $\{1, \ldots, n\}-\left\{i_{1}, \ldots, i_{b}\right\}$ is a $P$-vertex of $A$. Hence, $P_{v}(A)=b$.

\section{The optimal $P$-sets}

Let $T$ be a tree. We say that a set $P_{T}$ of $P(T)$ paths realizes $T$, if $P_{T}$ is a set of $P(T)$ vertex disjoint paths, occurring as induced subgraphs of $T$, that cover all vertices of $T$. In [4], the number $P(T)$ was studied. In particular the next two propositions appeared in that paper.

Proposition 3.1. (Proposition 2.2 in [4]) Let $T$ be a tree and $i$ be a vertex of $T$. Then

$$
P(T)-1 \leq P(T-i) \leq P(T)+1
$$

Proposition 3.2. (Proposition 3.3 in [4]) Let $T$ be a tree and $i$ be a vertex of $T$. Then $P(T-i)=P(T)+1$ if and only if $i$ is a vertex of degree two in every set of $P(T)$ paths that realizes $T$.

Remark 3.3. If $G$ is a forest (a union of trees) with components $T_{1}, \ldots, T_{l}$, then

$$
P(G)=\sum_{j=1}^{l} P\left(T_{j}\right)
$$

First we define a family of subsets of vertices of $T, \mathcal{A}_{T}$, that contains two kinds of sets necessary to determine a lower and an upper bound of the number of $P$-vertices in a matrix $A \in \mathcal{S}(T)$ with maximum nullity.

Definition 3.4. Let $T=(X, \mathcal{E})$ be a tree and $Y \subseteq X$. Let $c_{T}(Y)$ be the number of components of $T-Y$ and let 


$$
\mathcal{A}_{T}=\left\{R: R \subseteq X, c_{T}(R)=P(T)+|R|\right\}
$$

A set $R \in \mathcal{A}_{T}$ is called an optimal $P$-set of $T$.

Let $s_{T}$ be the number

$$
s_{T}=\min \left\{|R|: R \in \mathcal{A}_{T}\right\}
$$

and let $l_{T}$ be the number

$$
l_{T}=\max \left\{|R|: R \in \mathcal{A}_{T}\right\}
$$

A set $R \in \mathcal{A}_{T}$ of smallest cardinality is called an $s$-optimal $P$-set of $T$.

A set $R \in \mathcal{A}_{T}$ of largest cardinality is called an $l$-optimal $P$-set of $T$.

Example 3.5. Consider the following tree $T$ with $n=11$ vertices.

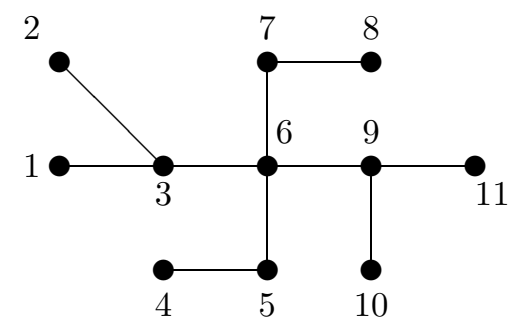

Note that $P(T)=3$ and no pendant vertex can be a $P$-vertex. The set $\mathcal{A}_{T}$ is the set

$$
\{\{6\},\{3,9\},\{3,6\},\{6,9\},\{3,6,9\},\{3,5,9\},\{3,7,9\},\{3,5,7,9\}\} .
$$

So,

- $\{6\}$ is an $s$-optimal $P$-set and $s_{T}=1$, and

- $\{3,5,7,9\}$ is an $l$-optimal $P$-set and $l_{T}=4$.

The next theorem shows another characterization of the elements of $\mathcal{A}_{T}$ that justifies the name of optimal $P$-sets.

Theorem 3.6. Let $T=(X, \mathcal{E})$ be a tree and $R$ be a subset of $X$. Then

$$
c_{T}(R) \leq P(T)+|R|
$$

Moreover, $c_{T}(R)=P(T)+|R|$ if and only if 
1. there exists $A \in \mathcal{S}(T)$ with $m_{A}(0)=M(T)$ and whose set of $P$-vertices is $R$, and

2. each component of $T-R$ is a path.

Proof. By Proposition 3.1,

$$
P(T-R) \leq P(T)+|R|
$$

Since

$$
c_{T}(R) \leq P(T-R)
$$

we conclude that

$$
c_{T}(R) \leq P(T)+|R|
$$

Now assume that $c_{T}(R)=P(T)+|R|$. Then $P(T-R)=c_{T}(R)$ and consequently, each component of $T-R$ is a path.

Let $A$ be a matrix in $\mathcal{S}(T)$ with $A(R)$ equal to the Laplacian matrix of $T-R$. Then by Theorem 2.4 we have $m_{A(R)}(0)=M(T-R)=P(T-R)=c_{T}(R)$, and

$$
M(T) \geq m_{A}(0) \geq m_{A(R)}(0)-|R|=c_{T}(R)-|R|=P(T)=M(T) .
$$

Therefore, $M(T)=m_{A}(0)=m_{A(R)}(0)-|R|$ and $R$ is a $P$-set of $A$.

Using Theorems 1.3 and 2.4 we know that the elements of $X-R$ are downer vertices of $A$. So, $R$ is the set of $P$-vertices of $A$.

Conversely, suppose that $R$ is a subset of $X$ and 1. and 2. hold. Using Theorem 1.3, $X-R$ is the set of downer vertices of $A$. Let $S$ be a component of $T-R$ and $x$ be a vertex of $S$. By Theorem 1.3, $x$ is a downer vertex of $A[S]$. This implies that $m_{A[S]}(0) \geq 1$. Since $S$ is a path then $m_{A[S]}(0)=1$.

So,

$$
c_{T}(R)=m_{A(R)}(0)=m_{A}(0)+|R|=P(T)+|R|
$$

Using the proof of last theorem we have the following corollary.

Corollary 3.7. Let $T$ be a tree and $R$ be an optimal $P$-set of $T$. Let $A \in \mathcal{S}(T)$ such that $A(R)$ is equal to the Laplacian matrix of $T-R$. Then $m_{A}(0)=M(T)$ and $R$ is the $P$-set of $A$.

\section{The optimal $P$-sets with smallest cardinality}

The purpose of this section is to establish proprieties of the optimal $P$-sets with smallest cardinality of $T$, where $T$ is a tree different from a path. 
Theorem 4.1. Let $T$ be a tree. Let $R$ be an optimal $P$-set and let $x \in R$. Then

1. $x$ is a vertex of degree two in every set of $P(T)$ paths that realizes $T$,

2. $d_{T}(x) \geq 2$,

3. if $d_{T}(x)=2$, then $R-x$ is an optimal $P$-set of $T$.

Proof. Since $R$ is a set of $\mathcal{A}_{T}$,

$$
c_{T}(R)=P(T)+|R|
$$

By Theorem 3.6, there exists $A \in \mathcal{S}(T)$ with $m_{A}(0)=M(T)$ and whose set of $P$-vertices is $R$, and each component of $T-R$ is a path. So, $P(T-R)=c_{T}(R)$. Consequently,

$$
P(T-R) \leq P(T-(R-x))+1 \leq P(T-x)+|R-x| \leq P(T)+|R|=P(T-R),
$$

and we conclude that $P(T-x)+|R|-1=P(T)+|R|$ and $P(T-R)=P(T-(R-x))+1$. By Proposition 3.2, $x$ is a vertex of degree two in every set of $P(T)$ paths that realizes $T$, and by Remark 3.3 and Proposition 3.2, $x$ is a vertex of degree two in every set of $P(T-(R-x))$ paths that realizes $T-(R-x)$. Thus, $d_{T}(x) \geq 2$ and $d_{T-(R-x)}(x) \geq 2$.

If $d_{T}(x)=2$ then $d_{T-(R-x)}(x)=2$ and $c_{T}(R)=c_{T}(R-x)+1$. Therefore, $c_{T}(R-x)=$ $c_{T}(R)-1=P(T)+|R|-1=P(T)+|R-x|$ and $R-x$ is an optimal $P$-set of $T$.

Using the last theorem we conclude the following corollary.

Corollary 4.2. Let $T$ be a tree that is not a path. Let $R$ be an s-optimal P-set of $T$. Then

$$
d_{T}(x)>2, \forall x \in R
$$

In [8] the authors defined a generalized star. Let $T$ be a tree that is not a path. We say that $S$ is a pendant generalized star of $T$ with central vertex $x$ if

- $S$ is a generalized star with central vertex $x$,

- $d_{T}(x)>2$,

- when we remove the vertices of $S$ from $T$, we obtain a nonempty tree, denoted by $T \backslash S$, and

- $x$ is adjacent to a vertex of $T \backslash S$. 
Example 4.3. Consider the following tree $T$ with $n=11$ vertices.

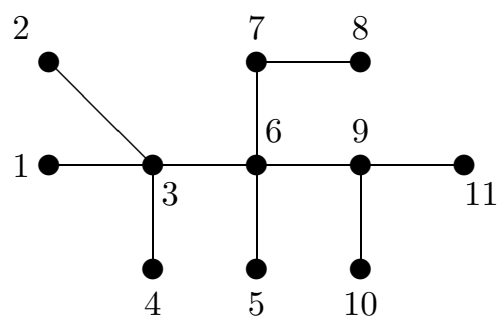

The subgraph of $T$ induced by vertices $1,2,3,4$ is a pendant generalized star of $T$ with central vertex 3 .

Proposition 4.4. Let $T$ be a tree. Then $s_{T} \leq P(T)-1$.

Proof. The proof is by induction on the number $l$ of vertices with degree greater than 2 . If $l=0$, then $T$ is a path, and so $s_{T}=0$ and $P(T)=1$. Therefore, $s_{T} \leq P(T)-1$.

Let $l \geq 0$, and assume that the result is true for trees with at most $l$ vertices of degree greater than two. Let $T_{1}$ be a tree with $l+1$ vertices of degree greater than two, and let $P_{T_{1}}$ be a set of $P\left(T_{1}\right)$ paths that realizes $T_{1}$. Let $S$ be a pendant generalized star of $T_{1}$ with central vertex $y$. Let $P$ be the path in $P_{T_{1}}$ where $y$ belongs. Then $d_{P}(y)=2$. In fact suppose that $d_{P}(y) \leq 1$. Since there are at least 2 components of $T_{1}-y$ that are paths, if $C_{1}$ is one of these paths that has no common vertices with $P$, then the concatenation of $C_{1}$ and $P$ results in a set, of $P\left(T_{1}\right)-1$ paths, that realizes $T_{1}$, which is impossible. By Proposition 3.2, $P\left(T_{1}-y\right)=P\left(T_{1}\right)+1$. By Remark 3.3, $P\left(T_{1}-y\right)=P\left(T_{1} \backslash S\right)+d_{T_{1}}(y)-1$. As $T_{1} \backslash S$ is a tree with at most $l$ vertices of degree greater than two, by induction, $s_{T_{1} \backslash S} \leq P\left(T_{1} \backslash S\right)-1$.

Let $R$ be an $s$-optimal $P$-set of $T_{1} \backslash S$. So,

$$
\begin{gathered}
c_{T_{1}}(R \cup\{y\})=c_{T_{1} \backslash S}(R)+d_{T_{1}}(y)-1=P\left(T_{1} \backslash S\right)+|R|+d_{T_{1}}(y)-1= \\
P\left(T_{1}-y\right)-d_{T_{1}}(y)+1+|R|+d_{T_{1}}(y)-1=P\left(T_{1}\right)+1+|R|=P\left(T_{1}\right)+|R \cup\{y\}| .
\end{gathered}
$$

Therefore, $R \cup\{y\}$ is an optimal $P$-set of $T_{1}$ and

$$
\begin{gathered}
s_{T_{1}} \leq|R|+1=s_{T_{1} \backslash S}+1 \leq P\left(T_{1} \backslash S\right)=P\left(T_{1}-y\right)-d_{T_{1}}(y)+1= \\
=P\left(T_{1}\right)-d_{T_{1}}(y)+2 \leq P\left(T_{1}\right)-1 .
\end{gathered}
$$

So, we have the result. 


\section{The optimal $P$-sets with largest cardinality}

As expected, the purpose of this section is to establish properties of the optimal $P$-sets with largest cardinality of $T$, where $T$ is a tree different from a path.

Theorem 5.1. Let $T=(X, \mathcal{E})$ be a tree. Let $R$ be an optimal $P$-set and let $x \in X-R$ such that $d_{T-R}(x)=2$, then $R \cup\{x\}$ is an optimal $P$-set of $T$.

Proof. Since $R$ is an optimal $P$-set then $c_{T}(R)=P(T)+|R|$. Because $d_{T-R}(x)=2$ then $c_{T}(R \cup\{x\})=c_{T}(R)+1$. Consequently, $c_{T}(R \cup\{x\})=P(T)+|R|+1=P(T)+|R \cup\{x\}|$ and $R \cup\{x\}$ is an optimal $P$-set of $T$.

The following corollary is the characterization of the components of $T-R$ when $R$ is an $l$-optimal $P$-sets of a tree and it is an easy consequence of last theorem.

Corollary 5.2. Let $T=(X, \mathcal{E})$ be a tree. Let $R$ be an l-optimal P-set. Then each component of $T-R$ is isomorphic to $K_{1}$ or $K_{2}$.

\section{Pendant generalized stars and optimal $P$-sets}

Let $T$ be a tree and $S$ be a pendant generalized star of $T$. In this section, we describe relations between some optimal $P$-sets of $T \backslash S$ and of $T$.

Lemma 6.1. Let $T=(X, \mathcal{E})$ be a tree. Let $S$ be a pendant generalized star of $T$ with central vertex $x$. Let $R \in \mathcal{A}_{T}$ such that $x \notin R$ and $d_{T}(z)>2, \forall z \in R$. Then $d_{T}(x)=3$.

Proof. Suppose $d_{T}(x)=s \geq 4$. Since, by Theorem 3.6, $T-R$ is a union of paths, $s \geq 4$ and $x \notin R$, then at least two neighbors of $x$ belong to $R$ and have degree 3 or more. We obtain a contradiction because one of these two neighbors of $x$ is a vertex of $S$ and has degree at most 2 .

Lemma 6.2. Let $T=(X, \mathcal{E})$ be a tree. Let $S$ be a pendant generalized star of $T$ with central vertex $x$. Let $w$ be the vertex of $T \backslash S$ adjacent to $x$. Let $R \in \mathcal{A}_{T}$ such that $x \notin R$ and $d_{T}(z)>2, \forall z \in R$. Then

1. $w \in R$ and $d_{T}(w) \geq 3$.

2. $w$ and $x$ are not in the same path for any set of $P(T)$ paths that realizes $T$.

3. $P(T)=P(T \backslash S)+1$.

4. $R$ is an optimal $P$-set of $T \backslash S$.

Proof. 1. Assume that $w \notin R$. Since by Theorem 3.6, $T-R$ is a union of paths, and $x \notin R$ and $d_{T}(x) \geq 3$, we conclude that there is a vertex $a_{2}$, adjacent to $x$, such that 
$a_{2}$ is not a vertex of $T \backslash S$ and $a_{2} \in R$. Since $d_{T}\left(a_{2}\right) \leq 2$ we obtain a contradiction. Then $w \in R$ and by hypothesis, $d_{T}(w) \geq 3$.

2. Let $S_{1}, \ldots, S_{P(T)}$ be a set of $P(T)$ paths that realizes $T$, and assume that $x, w \in S_{1}$. Since $x \notin R$, by Lemma $6.1, d_{T}(x)=3$. Let $a_{1}$ and $a_{2}$ be the vertices adjacent to $x$, different from $w$. Suppose that $d_{S_{1}}(x)=1$. Let $S_{2}$ be the path where $a_{1}$ belongs. So, $d_{S_{2}}\left(a_{1}\right)=1$ and $S_{1} \cup x \cup S_{2}, S_{3}, \ldots, S_{P(T)}$ is a set of $P(T)-1$ paths that realizes $T$. This is impossible, therefore $d_{S_{1}}(x)=2$. Let $a_{2}$ be the vertex of $S_{1}$. Assume that $a_{1}$ is a vertex of $S_{2}$. Let $S_{1}^{\prime}$ and $S_{1}^{\prime \prime}$ be the subpaths of $S_{1}-x$, and assume that $a_{2}$ is a vertex of $S_{1}^{\prime}$. Then $S_{1}^{\prime \prime}, S_{2} \cup x \cup S_{1}^{\prime}, S_{3}, \ldots, S_{P(T)}$ is a set of $P(T)$ paths that realizes $T$, and $w$ is a terminal vertex of $S_{1}^{\prime \prime}$. This is impossible by Theorem 4.1, because $w \in R$.

3. By Theorem 4.1, $w$ is a vertex of degree two in every set of $P(T)$ paths that realizes $T$. Using 2 . and the fact that $d_{T}(x)=3$ we conclude that $P(T)=P(T \backslash S)+1$.

4. Using 1., 3., then

$$
c_{T \backslash S}(R)=c_{T}(R)-1=P(T)+|R|-1=P(T \backslash S)+|R| .
$$

So, $R$ is an optimal $P$-set of $T \backslash S$.

Lemma 6.3. Let $T=(X, \mathcal{E})$ be a tree. Let $S$ be a pendant generalized star of $T$ with central vertex $x$. Let $R \in \mathcal{A}_{T}$ such that $x \in R$ and $d_{T}(z)>2, \forall z \in R$. Then $R-x$ is an optimal P-set of $T \backslash S$.

Proof. By Theorem 4.1, $x$ is a vertex of degree two in every set of $P(T)$ paths that realizes $T$. By Proposition 3.2, $P(T-x)=P(T)+1$. Since $P(T \backslash S)+d_{T}(x)-1=P(T-x)$, we conclude that

$$
\begin{gathered}
c_{T \backslash S}(R-x)=c_{T}(R)-d_{T}(x)+1=P(T)+|R|-d_{T}(x)+1= \\
=P(T \backslash S)+|R|-1=P(T \backslash S)+|R-x| .
\end{gathered}
$$

So, $R-x$ is an optimal $P$-set of $T \backslash S$.

The next theorem will be used in Section 7 to show that there are no gaps in the cardinality of $\mathcal{A}_{T}$, when $T$ is a tree.

Theorem 6.4. Let $T=(X, \mathcal{E})$ be a tree that is not a path, $R^{*}$ be an s-optimal P-set of $T$, and $U^{*}$ be an l-optimal $P$-set of $T$. Let $U$ be the set of all elements of $U^{*}$ that are vertices of degree greater than two of $T$ and let $Y$ be a subset of $X-R^{*}$ of largest cardinality that

- the subgraph spanned by vertices in $Y$ is the null graph, and

- $d_{T-R^{*}}(v)=2$, for all $v \in Y$. 
Then,

$$
|U| \leq s_{T}+|Y|
$$

Proof. Let $S$ be a pendant generalized star of $T$ with central vertex $x$. By Theorem 4.1, $U$ is an optimal $P$-set of $T$. We will divide the proof in four cases:

Case 1: If $x \notin R^{*}$ and $x \notin U$ then, by Lemma $6.2, R^{*}$ and $U$ are optimal $P$-sets of $T \backslash S$. Let $w$ be the vertex of $T \backslash S$ adjacent to $x$, by Lemma 6.2, then $w \in R^{*} \cap U$.

We repeat the process with $T \backslash S$ and

- if $d_{T \backslash S}(w)>2$, we consider the sets $R^{*}, U$ and $Y \cap(R \backslash S)$,

- if $d_{T \backslash S}(w)=2$, we consider the sets $R^{*}-w, U-w$ and $Y \cap(T \backslash S)$.

Case 2: If $x \notin R^{*}$ and $x \in U$.

Because $x \notin R^{*}$, by Lemma $6.1, d_{T}(x)=3$. Let $w$ be the vertex of $T \backslash S$ adjacent to $x$, by Lemma 6.2 , then $w \in R^{*}$. Let $P_{1}$ be the path of $T-R^{*}$ where $x$ belongs. Then $d_{P_{1}}(x)=2$. So $d_{T-R^{*}}(x)=2$. If $x \notin Y$, then at least one of the vertices of $P_{1}$ that are adjacent to $x$ is in $Y$. So, we associate the vertex $x$ to a vertex of $P_{1}$ that is in $Y$.

By Lemma 6.2 we have that $R^{*}$ is an optimal $P$-set of $T \backslash S$ and by Lemma $6.3, U-x$ is an optimal $P$-set of $T \backslash S$.

We repeat the process with $T \backslash S$ and

- if $d_{T \backslash S}(w)>2$, we consider the sets $R^{*}, U-x$ and $Y \cap(T \backslash S)$,

- if $d_{T \backslash S}(w)=2$, we consider the sets $R^{*}-w, U-x$ and $(Y \cup\{w\}) \cap(T \backslash S)$.

(Note that in this case, $d_{T}(w)=3$ and since $w \in R^{*}$ and $x \in U$, by Theorem 4.1 and Lemma 6.2, $w$ and $x$ are vertices of degree two in every set of $P(T)$ paths that realizes $T$ and they do not belong at the same of these paths. So, the vertices adjacent to $w$, different from $x$, are not in $Y$.)

Case 3: If $x \in R^{*}$ and $x \notin U$.

By Lemma 6.3 we have that $R^{*}-x$ is an optimal $P$-set of $T \backslash S$ and by Lemma 6.2, $U$ is an optimal $P$-set of $T \backslash S$ and $w \in U$.

We repeat the process with $T \backslash S$ and

- if $d_{T \backslash S}(w)>2$, we consider the sets $R^{*}-x, U$ and $Y \cap(T \backslash S)$,

- if $d_{T \backslash S}(w)=2$ and $w \in R^{*}$, we consider the sets $R^{*} \backslash\{x, w\}, U-w$ and $Y \cap(T \backslash S)$,

- if $d_{T \backslash S}(w)=2, w \notin R^{*}$ we associate the vertex $w$ with the vertex $x$, and we consider the sets $R^{*}-x, U-w$ and $(Y-w) \cap(T \backslash S)$.

Case 4: $x \in R^{*}$ and $x \in U$.

By Lemma 6.3, $R^{*}-x$ and $U-x$ are optimal $P$-sets of $T \backslash S$. We repeat the process with $T \backslash S$ and we consider the sets $R^{*}-x, U-x$ and $Y \cap(T \backslash S)$. 
Because each time we repeat the process and we remove a vertex of $U$ we also remove a vertex of $R^{*}$ or of $Y$, we conclude that $|U| \leq s_{T}+|Y|$.

\section{The number of $P$-vertices in a tree}

In this section, using the last sections, we determine an upper and a lower bound for the number of $P$-vertices of a matrix $A \in \mathcal{S}(T)$ with nullity $M(T)$, where $T$ is a tree. We also prove that if $b$ is an integer between these two bounds, then there is a matrix $A \in \mathcal{S}(T)$ such that $m_{A}(0)=M(T)$ and $A$ has $b P$-vertices.

Proposition 7.1. Let $T$ be a tree and let $A \in \mathcal{S}(T)$ with $m_{A}(0)=M(T)$. Then

$$
s_{T} \leq P_{v}(A) \leq l_{T}
$$

Proof. Let $B$ be the set of all $P$-vertices of $A$. If $T_{1}$ is a component of $T-B$ that is not a path, using the fact that $B$ is the set of $P$-vertices of $A$, by Theorem 1.1,

$$
m_{A\left[T_{1}\right]}(0) \leq 1
$$

Since we are assuming that $T_{1}$ is not a path there is a vertex $x$ of $T_{1}$ such that $d_{T_{1}}(x)>2$. Let $A_{1}^{\prime} \in \mathcal{S}\left(T_{1}\right)$ such that $m_{A_{1}^{\prime}}(0)=d_{T_{1}}(x)-1$ (for instance we can consider the Laplacian matrix associated to each component of $\left.T_{1}-x\right)$. Denote by $A^{\prime} \in \mathcal{S}(T)$ a matrix whose summands of $A^{\prime}(B)$ are the same of $A(B)$ except $A\left[T_{1}\right]$ that is replaced by $A_{1}^{\prime}$. Therefore, by the Cauchy interlacing Theorem

$$
m_{A^{\prime}}(0) \geq m_{A^{\prime}(B)}(0)-|B|>m_{A(B)}(0)-|B|=m_{A}(0)+|B|-|B|=M(T) .
$$

This is impossible. Then $T-B$ is a union of paths. By Theorem 3.6, $B$ is an optimal $P$-set of $T$. So, $s_{T} \leq|B|=P_{v}(A) \leq l_{T}$.

Theorem 7.2. Let $T=(X, \mathcal{E})$ be a tree and let $b$ be an integer such that

$$
s_{T} \leq b \leq l_{T}
$$

Then, there is a matrix $A \in \mathcal{S}(T)$ such that $m_{A}(0)=M(T)$ and $P_{v}(A)=b$.

Proof. Let $B$ be an $s$-optimal $P$-set of $T$. If all vertices of $T-B$ have degree at most one, let $Y=\emptyset$. If $T-B$ has at least one vertex of degree 2 , let $Y$ be a subset of $X-B$ of largest cardinality that

- the subgraph spanned by vertices in $Y$ is the null graph, and

- $d_{T-B}(v)=2$, for all $v \in Y$. 
By Theorem 5.1, $B \cup Y$ is an optimal $P$-set of $T$. Let $U^{*}$ be an $l$-optimal $P$-set of $T$ and let $U$ be the elements of $U^{*}$ that have degree greater than two in $T$. By Theorem 4.1, $U$ is an optimal P-set of $T$. By Theorem $6.4,|U| \leq|B \cup Y|$.

- If $|B| \leq b \leq|B \cup Y|$, since by Theorem 4.1, there is an optimal $P$-set, $F$, such that $B \subseteq F \subseteq(B \cup Y)$ with cardinality $b$, by Theorem 3.6 we have the result.

- If $|U| \leq b \leq\left|U^{*}\right|$, since by Theorem 5.1, there is an optimal P-set, $H$, such that $U \subseteq H \subseteq U^{*}$ with cardinality $b$, by Theorem 3.6 we have the result.

\section{Acknowledgements}

We thank Bryan Shader and C.M. da Fonseca for their comments in the previous version of this paper. We also thank the anonymous referee for many useful suggestions. This research was done while the second author visited the Universidade Nova de Lisboa. He would like to thank to Centro de Matemática e Aplicações, for their warm hospitality, and for all the conditions provided.

\section{References}

[1] D. Cvetkovic, P. Rowlinson, S.K. Simic, An Introduction to the Theory Graph Spectra, Student Texts, vol. 75, London Mathematical Society.

[2] Z. Du, C.M. da Fonseca, The acyclic matrices with a P-set of maximum size, Linear Algebra Appl. 468 (2015) 27-37.

[3] R. Fernandes, H.F. da Cruz, Sets of Parter vertices which are Parter sets, Linear Algebra Appl. 448 (2014) 37-54.

[4] R. Fernandes, On the multiplicity of an eigenvalue in a matrix whose graph contains exactly one cycle, Linear Algebra Appl. 422 (2007) 1-16.

[5] C.M. da Fonseca, On the eigenvalues of some tridiagonal matrices, J. Comput. Appl. Math. 200 (2007) 283-286.

[6] C.D. Godsil, Spectra of Trees, North-Holland Mathematics Studies, vol. 20, 1984.

[7] R.A. Horn, C.R. Johnson, Matrix Analysis, Cambridge University Press, New York, 1985.

[8] C.R. Johnson, A. Leal Duarte, C.M. Saiago, Inverse eigenvalue problems and lists of multiplicities of eigenvalues for matrices whose graph is a tree: the case of generalized stars and double stars, Linear Algebra Appl. 373 (2003) 311-330.

[9] C.R. Johnson, A. Leal Duarte, The maximum multiplicity of an eigenvalue in a matrix whose graph is a tree, Linear Multilinear Algebra 46 (1999) 139-144.

[10] C.R. Johnson, A. Leal Duarte, C.M. Saiago, The Parter-Wiener theorem: refinement and generalization, SIAM J. Matrix Anal. Appl. 25 (2003) 352-361.

[11] C.R. Johnson, A. Leal Duarte, C.M. Saiago, The structure of matrices with a maximum multiplicity eigenvalue, Linear Algebra Appl. 429 (2008) 875-886.

[12] C.R. Johnson, B.D. Sutton, Hermitian matrices eigenvalues multiplicities, and eigenvectors components, SIAM J. Matrix Anal. Appl. 26 (2004) 390-399.

[13] I.J. Kim, B.L. Shader, Non-singular acyclic matrices, Linear Multilinear Algebra 57 (2009) 399-407.

[14] C. Nelson, B.L. Shader, Maximal P-sets whose graph is a tree, Linear Algebra Appl. 485 (2015) $485-502$.

[15] S. Parter, On the eigenvalues and eigenvectors of a class matrices, J. Soc. Ind. Appl. Math. 8 (1960) $376-388$.

[16] G. Wiener, Spectral multiplicity and splitting results for a class of qualitative matrices, Linear Algebra Appl. 61 (1984) 15-29. 